REVISTA DE GESTADO ESECRETARIADO

MANAGEMENT AND ADMINISTRATIVE

PROFESSIONAL REVIEW

ISSN: 2178-9010
Revista GeSec

São Paulo, SP, Brasil

11(2), p. 164-189

maio/ago. 2020

DOI: http://dx.doi.org/10.7769/gesec.v11i2.1066

\title{
Os desafios de equipes multiculturais de trabalho: da liderança e influência da cultura nacional ao processo de aprendizagem organizacional
}

\section{The challenges of multicultural work teams: from leadership and the influence of national culture to the organizational learning process}

\author{
Fernanda Vicente Moraes ${ }^{1}$ \\ Janaína Maria Bueno ${ }^{2}$ \\ Carlos Roberto Domingues ${ }^{3}$
}

\begin{abstract}
Resumo
A formação de equipes multiculturais tem sido cada vez mais necessária e estimulada pelas organizações para construção de ambientes criativos e inovadores com intuito de responder a um contexto desafiador e em constante transformação (Bueno \& Freitas, 2015). Este estudo teve como objetivo analisar os desafios de três equipes multiculturais em subsidiárias de duas empresas multinacionais quanto às questões de liderança, cultura nacional e aprendizagem. Por meio de uma pesquisa qualitativa, do tipo descritiva, foi adotado o método de estudo de casos múltiplos, sendo a coleta de dados baseada em entrevistas semiestruturadas. A partir das três categorias definidas a priori, identificou-se que a liderança das equipes é do tipo única e vertical, o que reflete o pouco tempo de existência dessas equipes aliado à dificuldade que alguns indivíduos (brasileiros) sentem em se expressar em ambientes culturalmente diversos resultado de sua relação com traços culturais da gestão brasileira como distância do poder, postura de expectador, falta de planejamento e personalismo. As equipes aprenderam com base na interação cotidiana e troca de informações e opiniões, ressaltando a importância do ambiente multicultural e da adoção de algumas formas de registro do conhecimento que precisam ser aperfeiçoados e expandidos. Conclui-se que as equipes multiculturais estudadas foram responsáveis pela geração de novos conhecimentos para as empresas, obtiveram resultados além do esperado e ajudaram a projetar a carreira de seus membros, evidenciando que esta experiência pode ser enriquecedora para os profissionais envolvidos e para as organizações, sendo válido investir no amadurecimento do processo de liderança, entendimento das diferenças culturais e aperfeiçoamento do processo de aprendizagem organizacional.
\end{abstract}

Palavras-chaves: Equipes Multiculturais. Ambiente Multicultural. Gestão Intercultural. Aprendizagem Organizacional.

\footnotetext{
Abstract

The creation of multicultural teams has been increasingly necessary and stimulated by organizations to build creative and innovative environments in order to respond to a challenging and constantly changing context (Bueno \& Freitas, 2015). This study aimed to analyze the challenges of three multicultural teams in subsidiaries of two multinational companies regarding the issues of leadership, national culture and learning. Through a qualitative, descriptive research, the method of multiple case study was adopted, and data collection was based on semi-structured interviews. From three categories

${ }^{1}$ Bacharel em Administração.

${ }^{2}$ Pós-doutora em Administração, Doutora em Administração, Docente no Programa de Pós-graduação em Administração da Universidade Federal de Uberlândia (UFU).

${ }^{3}$ Doutor em Administração, Docente do Programa de Pós-graduação em Administração da UFU.
} 
defined a priori (Leadership; Influence of National Culture; and Organizational Learning), it was identified that the type of leadership of the teams is unique and vertical, which reflects the short time of existence of these teams combined with the difficulty of some individuals (Brazilians) have to express feelings and opinions in culturally diverse environment. This difficulty results from the cultural traits of Brazilian management such as power distance, expectant posture, lack of planning and personalism. The results showed that the learning process is based on daily interaction and exchange of information and opinions, emphasizing the importance of the multicultural environment and the adoption of some ways of knowledge recording that need to be improved and expanded. Finally, we conclude that multicultural teams studied were responsible for generating new knowledge to companies, achieved results beyond expectations and helped design the career of its members, showing that this experience can be enriching for professionals involved and the organizations, being valid invest in the maturing of the leadership process, understanding of cultural differences and improvement of the organizational learning process.

Keywords: Multicultural Teams. Multicultural environment. Intercultural management. Organizational Learning.

\section{Introdução}

As equipes multiculturais podem ser entendidas como grupos de trabalho compostos por pessoas de diferentes nacionalidades que desenvolvem atividades com objetivo comum (Adler \& Gundersen, 2008) na forma de projetos, por exemplo (Ribeiro, 2010), a fim de atender às estratégias organizacionais. As equipes culturalmente diversas podem ser formadas por membros atuantes em um mesmo local, chamadas comumente de tradicionais, com profissionais expatriados e locais. Podem também ser formadas por membros espalhados em diferentes unidades da empresa ou de várias empresas, chamadas de equipes virtuais, sendo seus contatos, geralmente, mediados por sistemas de informação e comunicação (Krumm, Terwiel, \& Hertel, 2013).

As equipes multiculturais, cada vez mais, são criadas como um elemento central para os resultados das empresas, sendo vistas como um recurso estratégico no processo de internacionalização, no desenvolvimento de ambientes criativos e inovadores, na formação de novos gestores e até mesmo na sistematização de processos em suas diferentes unidades (Adler \& Gundersen, 2008; Morgeson et al, 2010; Blanche \& Dupuis, 2019). Elas podem oferecer diferentes benefícios para as empresas, já que um grupo heterogêneo (em ideias, nacionalidades, gêneros, formação, entre outras características) responde positivamente a um ambiente desafiador que demanda por criatividade e inovação, podendo ser capaz de ter melhor desempenho e resultados comparados com equipes homogêneas, conforme já demonstrado por diferentes pesquisas (Bueno \& Freitas, 2015; Mannix \& Neale, 2016).

Apesar das equipes multiculturais possuírem potencial para terem alto desempenho, elas apresentam desafios para seu bom gerenciamento e consequentemente realização de 
tarefas de acordo com as expectativas (Bueno \& Freitas, 2015). Um dos pontos cruciais para o bom desempenho de qualquer equipe é, segundo Heinz (2014), o estabelecimento de uma comunicação, no mínimo, razoável sendo este um dos pontos mais críticos e que merece gerenciamento e atenção. Não somente a linguagem verbal pode ser um desafio na gestão, mas também a linguagem não verbal, destacando a importância dos diferentes contextos de comunicação (Hall, 1976). Outro ponto delicado é como lidar com a diversidade cultural que, apesar de proporcionar diferentes perspectivas e possibilitar soluções diferentes para um mesmo problema, demanda por tempo, paciência e empatia (Adler \& Gundersen, 2008; Blanche \& Dupuis, 2019).

Dentro do exposto, o objetivo desta pesquisa foi analisar, em duas empresas multinacionais, os desafios de três equipes multiculturais de trabalho tendo como parâmetros as questões sobre liderança, a influência da cultura nacional e o processo de aprendizagem organizacional.

Este trabalho justifica-se, sob o ponto de vista teórico e empírico, pela escassa literatura sobre o tema nas bases de dados acadêmicas brasileiras que retrate o trabalho deste tipo de equipe (Bueno \& Freitas, 2018) e pela contribuição com a discussão sobre os ganhos em longo prazo para as organizações e para os indivíduos envolvidos.

\section{Referencial Teórico}

\subsection{Equipes multiculturais e liderança}

A busca pelo equilíbrio entre a integração global e a capacidade de resposta local tem se acelerado e sido o desafio das estratégias de empresas multinacionais (Zander \& Butler, 2010) ou em processo de internacionalização. E um dos caminhos para o seu alcance passa pelos processos de mobilidade internacional, como a expatriação, e pela consequente criação e desenvolvimento de equipes multiculturais de trabalho. Conforme Connaughton e Shuffler (2007), equipes que possuem diversidade geográfica, temporal e cultural estão se tornando, cada vez mais, presentes e relevantes em muitos setores.

De acordo com Krumm et al (2013), o contato entre culturas e a formação de equipes multiculturais acontecem não somente pela mobilidade internacional. Meios de comunicação como vídeos e teleconferências, sistemas de gestão, entre outras ferramentas de tecnologia e comunicação são usados para viabilizar a realização da tarefa proposta para a equipe, de forma virtual. Para Heinz (2014), as equipes multiculturais virtuais são um tipo de equipe que 
vem crescendo nos últimos anos e se popularizando porque facilitam a criação de projetos menores e não se constituem em processos caros e demorados como a expatriação de profissionais. Conforme Algesheimer et al (2011), alianças internacionais e estratégias offshoring - realocação de um processo de negócio em outro país - crescem cada vez mais e essas estratégias podem necessitar do trabalho de equipes intraorganizacionais e/ou interorganizacionais tradicionais e virtuais. Segundo Algesheimer, Dholakia e Gurau (2011), esta segunda configuração pode ser mais vantajosa para as empresas, pois reduz custos de viagens ou custos e riscos relacionados à expatriação. Contudo, ela gera mais desafios para os gestores e tende a ser mais limitada do ponto de vista de interações e aprendizado.

Uma das principais vantagens apontada na literatura para as equipes multiculturais, no geral, é conhecida como “o valor da diversidade" (Mannix \& Neale, 2016) baseada nos estudos de L. Hoffman e N. Maier, em seu artigo de 1961 intitulado "Quality and acceptance of problem solutions by members of homogeneous and heterogeneous groups", onde estudaram pequenos grupos heterogêneos e a interpretação do comportamento destes grupos resultou na afirmação de que a diversidade cria valor e benefícios para as equipes e até os desafios fazem com que a interação melhore. O estudo sugere, ainda, que em grupos com diversidade é esperado que se tenham mais conhecimento, expertise e perspectivas do que em grupos homogêneos. Esses fatores devem facilitar o desempenho da equipe quando se trata de tarefas complexas que envolvem discussão de perspectivas.

Conforme Adler e Gundersen (2008), as equipes que são culturalmente diversas possuem potencial para produtividade acima da média comparadas às equipes homogêneas, pois possuem perspectivas e experiências diferentes o que facilita o surgimento de diferentes ideias. Bueno e Freitas (2018) argumentam que o potencial para produtividade superior de equipes culturalmente diversas é alto; elas possuem o 'fôlego de recursos', insights, perspectivas e experiências diferenciadas que podem facilitar a criação de novas e melhores soluções. Para Zander e Butler (2010), elas são vistas como a fonte de iniciativas criativas para as organizações, agindo como 'cola' entre as fronteiras nacionais e culturais. No entanto, estas equipes não conseguem atingir seu potencial caso não sejam bem gerenciadas. Além disso, de acordo com as autoras, uma variedade de estudos constata que a maioria das equipes falha ao dar o retorno esperado e apresenta dificuldade em cumprir seus objetivos.

Para Heinz (2014), é preciso prestar atenção, especialmente, em situações conflituosas que necessitam mudanças e adequações nas equipes culturalmente diversas. Caso os membros da equipe não sejam capazes de enxergá-las e fazê-las, esta diversidade cultural pode trazer problemas para a gestão, baixar a produtividade e ainda deteriorar as relações estabelecidas. 
Com sua pesquisa, Stahl, Maznevski, Voigt \& Jonsen (2010) demonstraram que as equipes multiculturais podem acarretar em perdas do ponto de vista dos processos organizacionais com a diminuição da integração social e simultâneo aumento de conflitos. Por outro lado, elas podem gerar ganhos de processos com o aumento da criatividade e a satisfação de seus membros evidenciando, assim, que a gestão das equipes e dos processos inerentes ao seu trabalho são muito importantes.

Zander e Butler (2010), com base em pesquisas sobre liderança, apontam que ela está se tornando um fenômeno compartilhado nas organizações. Mesmo sendo tema de diversos estudos, especificamente a liderança em equipes multiculturais tem sido um tema pouco desenvolvido na literatura acadêmica. Para tentar suprir esta lacuna e ajudar na performance de equipes multiculturais, as autoras propuseram um modelo de escolha de liderança neste contexto ancorado em duas dimensões: i) atividades de liderança focada versus distribuída (em práticas e funções específicas) e ii) autoridade de liderança vertical versus horizontal (autoridade de tomada de decisão específica). A partir daí, são descritos quatro modos de liderança: liderança única; liderança em pares; liderança rotativa; e liderança compartilhada. Estas dimensões e modos de liderança podem ser observados na Figura 1:

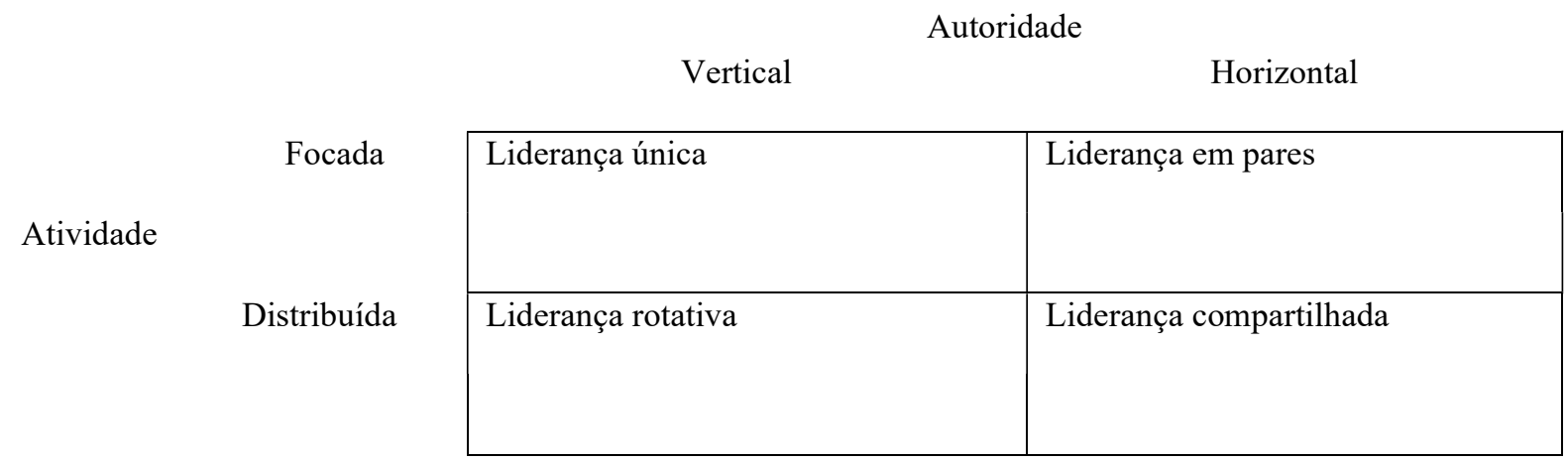

Figura 1. Tipologia de Modos de Liderança

Fonte: Adaptado de Zander e Butler (2010, p. 260).

De acordo com esta tipologia, a autoridade vertical é aquela que atende às atividades focadas de uma única equipe, sendo que a autoridade de tomada de decisão e as práticas e funções de liderança são todas centradas no mesmo indivíduo. Alguns estudos mostraram que, em equipes com alta interdependência de tarefas e altas demandas de coordenação, a liderança única (voltada para capacitação, monitoramento, feedback e estímulo à participação) estava positivamente relacionada ao desempenho e aprendizado das equipes multiculturais. Já a liderança única combinada com a autoridade horizontal (em pares) é indicada quando ambos 
os líderes têm influência e autoridade de tomada de decisão, mas em atividades diferentes, compartilhando a responsabilidade de liderar o grupo (Zander \& Butler, 2010).

$\mathrm{Na}$ liderança de equipe rotativa, as funções e práticas de liderança são distribuídas para um grupo menor de membros da equipe. A estrutura de liderança vertical sugere que esses membros da equipe não compartilham a autoridade de tomada de decisão. Ao invés disso, ela é deslocada de um indivíduo para outro. Esse modo de liderança, segundo Zander e Butler (2010), pode ser observado em equipes de ação extrema onde membros altamente qualificados cooperam para executar tarefas imprevisíveis e urgentes, ao mesmo tempo em que lidam com mudanças frequentes na composição da equipe. Enquanto que, na liderança compartilhada, os membros da equipe não apenas participam, pois, a liderança provém do grupo. Para construir tal capacidade de liderança no nível da equipe, os indivíduos interagem com o objetivo de realização de um trabalho compartilhado, de aprendizagem e desenvolvimento conjunto onde a cooperação torna-se particularmente importante, complementam as autoras.

Completa o modelo a análise de Zander e Butler (2010) a questão da composição da equipe que é baseada em duas abordagens: i) linhas de fragmentação e ii) definição de status, como apresentado na Figura 2. Dado que uma forte relação entre a composição da equipe e o seu desempenho foi estabelecida empiricamente, a formação da equipe pode ser considerada uma das funções mais importantes para os líderes (Morgeson et al., 2010).

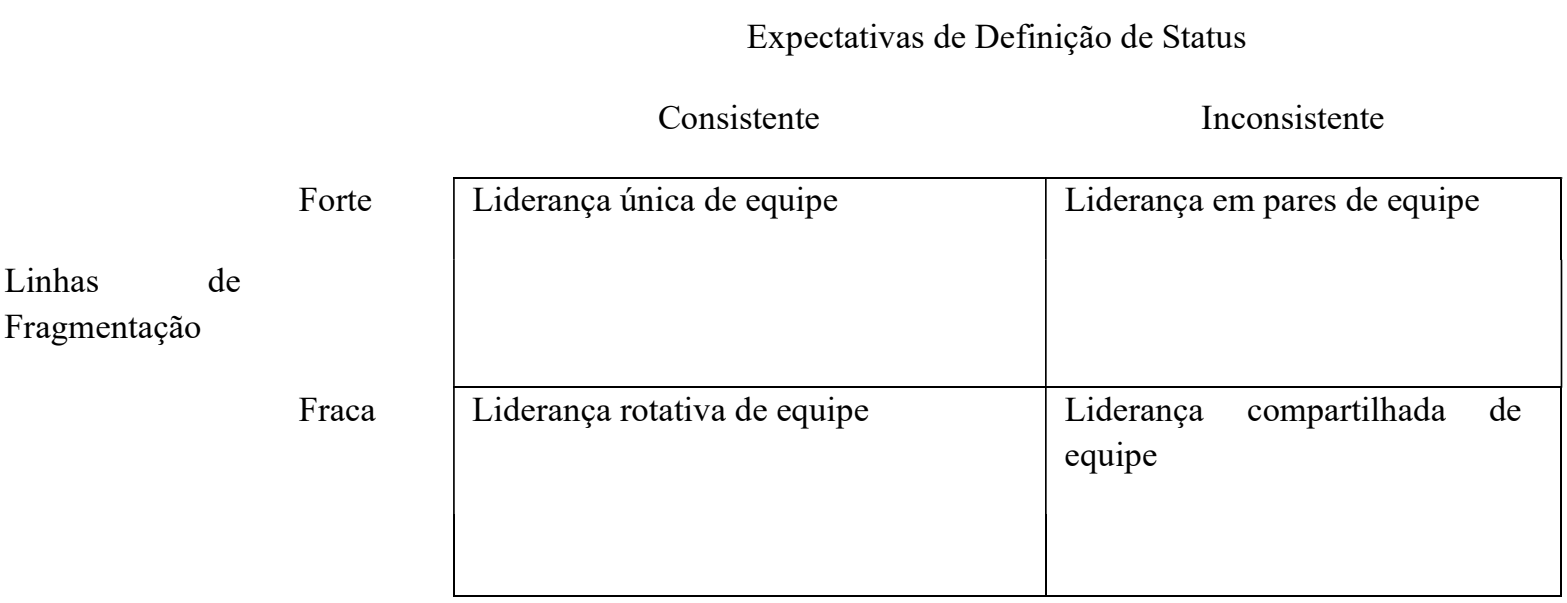

Figura 2. Modos de Liderança em Equipe Baseados na Composição da equipe Fonte: Adaptado de Zander e Butler (2010, p. 263).

Para as autoras, o número de subgrupos e a força correspondente da linha de fragmentação dependem do alinhamento dos atributos individuais aparentes aos membros do grupo. Quanto mais atributos demográficos dos membros do grupo estiverem correlacionados, menor o número de subgrupos e mais homogêneos, mais fracas as linhas de fragmentação. 
Quando estas linhas de fragmentação são fortes, o poder e a rivalidade entre subgrupos aumentam. Os exemplos incluem sotaques, volume da fala, cor da pele, sexo e formas de se vestir, entre outros. Indicações categóricas fornecem informações sobre quem é cada indivíduo e podem ser consistentes nas expectativas criadas (demandando a liderança única de equipe e a liderança compartilhada de equipe) ou inconsistentes (que levam à liderança em pares e liderança rotativa da equipe). Indicações mais inconsistentes com as expectativas levam a situações mais igualitárias, enquanto indicações mais consistentes com as expectativas tendem a formação de grupos mais hierárquicos (Zander \& Butler, 2010).

\subsection{A influência da cultura nacional}

A cultura é vista como uma força crítica de localização (Pudelko, Carr \& Henley, 2007), contribuindo para o aumento da complexidade dos negócios além das fronteiras nacionais. Para as empresas, as equipes multiculturais podem ser uma maneira eficiente de lidar com as especificidades locais e, ao mesmo tempo, alcançar a coordenação global.

A cultura, na concepção clássica de Schein (2009), é um fenômeno que cerca todos os seres humanos acontecendo constantemente e sendo criada a partir das interações realizadas, como um conjunto de regras, rotinas, costumes e estruturas que regem um grupo de pessoas e que orientam o comportamento daqueles pertencentes à comunidade. Para Chevrier (2009), é possível estudar a cultura em diferentes níveis resultando em maior ou menor grau de detalhes e informação adicional como, por exemplo, observar a ação de empresas multinacionais sob o ponto de vista da cultura nacional, onde o desafio é definir em cada nível uma abordagem consistente sobre o que é compartilhado ou não. A autora também discute o conceito de cultura política nacional que ajuda a compreender como os países desenvolvem suas referências para lidar com as ameaças compartilhadas que mais amedrontam. Tais referências podem ajudar as empresas e indivíduos a trabalhar em ambientes e equipes multiculturais.

A comunicação também é um importante aspecto a ser considerado no estudo de diferentes culturas e, para Adler e Gundersen (2008), a comunicação intercultural envolve um processo complexo, multifacetado e dinâmico no qual os indivíduos trocam e compartilham significados. Hall (1976) foi um dos pioneiros ao abordar as diferenças na comunicação entre culturas de alto e de baixo contexto sendo que, para o autor, nas culturas de alto contexto a comunicação, em grande parte, ocorre por meio de elementos não verbais ou escritos, como a linguagem corporal e a postura. Já nas culturas de baixo contexto, a comunicação se dá primordialmente pelas palavras escritas ou faladas.

Revista de Gestão e Secretariado (GeSec), São Paulo, SP, 11(2), maio/ago., 2020, p. 164-189. 
Do ponto de vista da gestão de empresas, durante as décadas de 1980 e 1990 houve um crescimento expressivo de estudos sobre a mobilidade internacional e seus efeitos para as empresas e indivíduos. Eles trouxeram contribuições para a comparação de diferentes culturas com base em algumas dimensões encontradas, como é o caso de Hofstede (1984) e sua pesquisa pioneira com subsidiárias da IBM em diferentes países. Sua pesquisa foi um marco sobre o papel da cultura nacional na explicação das diferenças de atitudes e valores em relação ao trabalho. Geralmente, outros fatores eram tidos como mais importantes como a posição na organização, a profissão, idade ou gênero. Hofstede (1984) identificou e validou dimensões que diferenciam e aproximam comportamentos entre profissionais no ambiente de trabalho, dependendo da sua cultura nacional, sendo estas dimensões: individualismo/coletivismo; distância do poder; fuga da incerteza; sociedade mais feminina/masculina, orientação de longo/curto prazo.

O Brasil estava entre os países pesquisados por Hofstede (1984) e os resultados apontaram para uma sociedade de características coletivistas, por grande distância de poder, busca por evitar incertezas e orientação de curto prazo. Seguindo a linha deste autor aliado a outros estudos sobre a cultura brasileira, Chu (2011) analisa a cultura brasileira como sendo plural e multifacetada e que influencia os modelos de gestão. A autora discute alguns traços da cultura brasileira que refletem diretamente no modo como gestores brasileiros administram situações e tomam decisões nas organizações como: o jeitinho, a desigualdade de poder e hierarquia, a aversão ao conflito, a flexibilidade, a postura de expectador, a plasticidade, a feminilidade, o personalismo, a falta de planejamento e o formalismo. A figura 3 apresenta uma síntese dos traços culturais citados pela autora:

\begin{tabular}{|c|l|}
\hline Traço & \multicolumn{1}{c|}{ Característica } \\
\hline Jeitinho & $\begin{array}{l}\text { Comportamento que tem como objetivo amenizar e harmonizar as regras do cotidiano, } \\
\text { sendo uma maneira de suavizar as relações impessoais, tornando-as o mais pessoal } \\
\text { possível. }\end{array}$ \\
\hline $\begin{array}{c}\text { Desigualdade } \\
\text { de poder e } \\
\text { hierarquia }\end{array}$ & $\begin{array}{l}\text { A desigualdade de poder permeia a sociedade brasileira, isso faz com que o status, a } \\
\text { hierarquia e as relações de autoridade tenham grande importância para os brasileiros. }\end{array}$ \\
\hline $\begin{array}{c}\text { Aversão ao } \\
\text { conflito }\end{array}$ & $\begin{array}{l}\text { Este traço se mostra através da maneira como os brasileiros harmonizam os grupos os } \\
\text { quais pertencem, evitando constrangimentos e divergências. }\end{array}$ \\
\hline $\begin{array}{c}\text { Flexibilidade } \\
\text { Postura de } \\
\text { expectador }\end{array}$ & $\begin{array}{l}\text { Esta característica se revela a partir da rapidez na adaptação e na criatividade diante de } \\
\text { situaço esperadas. } \\
\text { organizações, é um reflexo do mandonismo existente na sociedade brasileira. }\end{array}$ \\
\hline
\end{tabular}




\begin{tabular}{|c|l|} 
Plasticidade & $\begin{array}{l}\text { Este traço é percebido pela admiração e pelo gosto do brasileiro às práticas estrangeiras, } \\
\text { preferindo na maioria das vezes conceitos e produtos desenvolvidos em outros países do } \\
\text { que aqueles nacionais. }\end{array}$ \\
\hline Feminilidade & $\begin{array}{l}\text { Orientação para comportamentos que apreciam o bem-estar do outro, valorizando o } \\
\text { relacionamento entre as pessoas. Revela-se na ação por intuição e pelo zelo em se } \\
\text { relacionar bem com os superiores. }\end{array}$ \\
\hline Planejamento & $\begin{array}{l}\text { Demostra a importância dada para as relações construídas e interesses pessoais em } \\
\text { tetrimento dos interesses do grupo. Em ambientes organizacionais, os líderes e liderados } \\
\text { constroem relações que vão além da empresa. } \\
\text { curto prazo. }\end{array}$ \\
\hline Formalismejamento é vista como um traço histórico ligado a economia brasileira, por \\
\hline $\begin{array}{l}\text { Está intimamente ligado ao fato do brasileiro ter aversão a ambientes incertos, o } \\
\text { formalismo se mostra através da grande quantidade de regras e leis existentes, que na } \\
\text { maioria das vezes não condiz com a realidade. }\end{array}$ \\
\hline
\end{tabular}

Figura 3. Traços da Cultura Brasileira que Afetam a Gestão de Organizações

Fonte: Adaptado de Chu (2011, p. 23-26).

Para Chu (2011), as equipes multiculturais e o advento da globalização contribuíram muito para a reformulação dos modelos gerenciais amenizando a influência da cultura nacional. Apesar de não ser possível extingui-la, os diferentes modelos de gestão nacionais tendem a incorporar práticas de outros lugares e formar o chamado modelo "Glocal" que mescla as práticas tradicionais da cultura nacional com as práticas estrangeiras que são adaptadas ao contexto enfrentado pelos gestores. É importante ressaltar que o modelo "Glocal" ressignifica as práticas já estabelecidas, sintetizando e transformando o estilo antigo com o novo. Na mesma linha, Blanche e Dupuis (2019) argumentam que há uma boa chance dos indivíduos não se comportarem mais tão de acordo com o que escreveram os autores mais antigos sobre cada cultura nacional, pois eles precisaram e precisam se adaptar e se ajustar às diferentes culturas das pessoas com quem se relacionam, principalmente no ambiente de trabalho, para poderem trabalhar e cooperar com colegas e superiores a fim de atingir seus objetivos.

\subsection{Aprendizagem organizacional}

Os indivíduos que fazem parte de equipes multiculturais adquirem know-how específico sobre projetos e tarefas que desenvolvem e, como grupo, criam e disseminam conhecimento coletivo. A aprendizagem organizacional pode ser definida como um processo dinâmico de criação, aquisição e integração de conhecimento destinada ao desenvolvimento de recursos e capacidades que contribuem para a melhora da performance organizacional 
(Lopez, Peón \& Ordáz, 2015). Para Fleury e Fleury (2001), “as organizações podem não ter cérebros, mas têm sistemas cognitivos e memórias; desenvolvem rotinas, procedimentos relativamente padronizados para lidar com os problemas internos e externos. Estas rotinas vão sendo incorporadas, de forma explícita ou inconsciente, na memória organizacional” (p. 5).

Garvin, Edmondson e Gino (2008) afirmam que a aprendizagem organizacional ocorre mais facilmente em organizações que são plurais, ou seja, aquelas que possuem diversidade na formação de suas equipes, sendo a diversidade relacionada a gênero, cultura, idade, etnias, entre outros. Além disso, as organizações que proporcionam um clima aberto às novas ideias e contam com líderes que as incentivem, são receptivos e compreensivos com novos pontos de vistas, são organizações que conseguem aprender de forma melhor e mais eficaz. Para Blanche e Dupuis (2019), nas interações entre os membros das equipes multiculturais são compartilhadas práticas, valores e conhecimento. E o compartilhamento do conhecimento é visto como o ponto central da dinâmica intercultural.

O problema, segundo Bueno e Freitas (2018), é que nem sempre as organizações dão a atenção devida ao processo de aprendizagem organizacional quando se trata de equipes multiculturais. De acordo com Strauhs, Pietrovski, Santos, Carvalho, Pimenta e Penteado (2012), para que a aprendizagem organizacional seja realmente uma vantagem competitiva para a empresa é preciso que o conhecimento gerado esteja documentado e as gerações futuras possam acessá-lo de forma prática e rápida. Nesse sentido, Bueno e Freitas (2018) sugerem que o registro e a sistematização das práticas interculturais podem ajudar a repensar políticas e práticas específicas do ambiente e do trabalho multicultural, podendo aumentar ganhos individuais e organizacionais.

O foco nas atividades mentais individuais como o elemento central no processo de aquisição de informações reflete-se na construção da memória organizacional. Entretanto, as interpretações de problemas e as respectivas soluções são particulares do indivíduo e para que essas informações não sejam perdidas mesmo quando indivíduos chaves deixam as organizações é necessário que essas interpretações e soluções sejam compartilhadas (Takeuchi \& Nonaka, 2008). Conforme Garvin, Edmondson e Gino (2008), um ambiente que apoie a aprendizagem deve oferecer segurança psicológica, ou seja, para aprender os indivíduos não podem sentir medo, desprezados e marginalizados quando discordam de seus gestores, fazem perguntas e até mesmo erram ou quando demonstram seu ponto de vista.

Silva e Burger (2017) comentam que, cada vez mais, as organizações estão preocupadas com o gerenciamento do conhecimento adquirido, visto que este pode levar a uma importante vantagem competitiva e incentivar a inovação, passando assim a ser parte 
fundamental do ambiente corporativo. O conhecimento é multidimensional, sendo assim, gerencia-lo não é uma prática simples, muito menos fácil de ser implementada nas organizações. Portanto, é necessário que os gestores busquem modos para obter novos conhecimentos e para compartilhá-los, além de analisar sua aplicabilidade diante dos projetos, equipes e a organização em si.

Conforme Strauhs et al (2012), a gestão do conhecimento é capaz de reunir condições e criar uma estrutura apropriada envolvendo documentos, dados e informações físicos e/ou digitais e, a partir disso, colocar em prática a gestão dos mais variados conhecimentos pessoais ou técnicos existentes nas organizações. Os desafios para os gestores, de acordo com Silva e Burger (2017), é conseguir estabelecer áreas para direcionar os esforços, estabelecer metas, estimular os indivíduos e fomentar uma cultura voltada para a inovação e o aprendizado.

\section{Procedimentos metodológicos}

A pesquisa foi do tipo descritiva, de abordagem qualitativa, com o procedimento técnico de estudo de caso realizado em 3 equipes multiculturais de 2 empresas - uma delas é uma multinacional estrangeira e a outra é uma empresa brasileira em processo de internacionalização. O critério de escolha das empresas foi por conveniência, ou seja, não probabilístico, em duas empresas que possuem unidades em uma cidade de médio porte situada em Minas Gerais. Estas duas empresas (denominadas neste trabalho de empresa Alfa e Beta) possuem equipes de trabalho compostas por profissionais brasileiros e estrangeiros que interagem cotidianamente tanto de forma tradicional como em interações virtuais.

A coleta de dados foi realizada por meio de entrevistas semiestruturadas seguindo um roteiro pré-definido que foi desenvolvido com base no referencial teórico. Na Empresa Alfa foram entrevistados 4 indivíduos que trabalham em uma mesma equipe multicultural. $\mathrm{Na}$ Empresa Beta, foram entrevistados 3 indivíduos que integram 2 equipes distintas, um dos entrevistados foi líder das duas equipes multiculturais. O critério de escolha dos entrevistados se deu pelo método da bola de neve, Vinuto (2014) afirma que este método não probabilístico pressupõe que existe uma ligação entre os indivíduos, dessa forma, para se iniciar o estudo deve-se ter pelo menos um membro conhecido que é chamado de "semente" da amostra e este realiza indicações de outros indivíduos que se encaixam no grupo. Neste estudo, o método da bola de neve foi utilizado a partir do conhecimento de dois membros sementes que foram contatados e indicaram outros profissionais membros de equipes culturalmente diversas. 
As entrevistas foram gravadas e, posteriormente, transcritas na íntegra para que pudesse ser feita a análise de conteúdo, conforme preceitos de Bardin (2009).

A figura 4 apresenta uma síntese das informações sobre os entrevistados:

\begin{tabular}{|c|c|c|c|}
\hline Empresa & Equipe & Entrevistado & Perfil \\
\hline Alfa & Equipe 1 & Alfa E1-1 & $\begin{array}{c}\text { Homem, } 30 \text { anos, brasileiro, com experiência multicultural anterior, } \\
\text { especialista em Contas a Pagar. }\end{array}$ \\
\hline Alfa & Equipe 1 & Alfa E1-2 & $\begin{array}{l}\text { Mulher, } 32 \text { anos, brasileira, breve experiência em equipes } \\
\text { multiculturais, analista de Compras. }\end{array}$ \\
\hline Alfa & Equipe 1 & Alfa E1-3 & $\begin{array}{c}\text { Homem, } 38 \text { anos, brasileiro, sem nenhuma experiência anterior } \\
\text { com equipes culturalmente diversas, analista de dados. }\end{array}$ \\
\hline Alfa & Equipe 1 & Alfa E1-4 & $\begin{array}{c}\text { Homem, } 57 \text { anos, brasileiro, sem experiências relevantes anteriores } \\
\text { com equipes multiculturais, líder funcional de P2O (Procurement to } \\
\text { Pay) e I2P (Invoice to Pay) no Brasil. }\end{array}$ \\
\hline Beta & $\begin{array}{l}\text { Equipe } 2 \\
\quad \text { e } 3\end{array}$ & Beta E2/3-5 & $\begin{array}{c}\text { Homem, } 51 \text { anos, brasileiro, diretor de inovação na empresa Beta, } \\
\text { possui experiência prévia como líder e liderado em equipes } \\
\text { multiculturais. }\end{array}$ \\
\hline Beta & Equipe 2 & Beta E2-6 & $\begin{array}{l}\text { Mulher, } 25 \text { anos, colombiana, analista de inovação, participou do } \\
\text { projeto para internacionalização da empresa por } 6 \text { meses e já } \\
\text { possuía experiência anterior em equipes multiculturais. }\end{array}$ \\
\hline Beta & Equipe 3 & Beta E3-7 & $\begin{array}{c}\text { Homem, } 26 \text { anos, equatoriano, analista de inovação por } 6 \text { meses, } \\
\text { participou vários projetos ligados a inovação da companhia, já } \\
\text { possuía breve experiência com equipes multiculturais. }\end{array}$ \\
\hline
\end{tabular}

Figura 4. Perfil dos entrevistados

Fonte: Dados da pesquisa.

Durante o processo de análise dos dados coletados, alguns entrevistados foram contatados uma segunda vez com a finalidade de sanar pontos de dúvida que surgiram.

\section{Apresentação e análise dos resultados}

\subsection{Objetivos e composição das equipes multiculturais}

As equipes multiculturais da empresa Alfa e Beta estão caracterizadas nas Figuras 5 e 6, considerando os seus principais aspectos. 


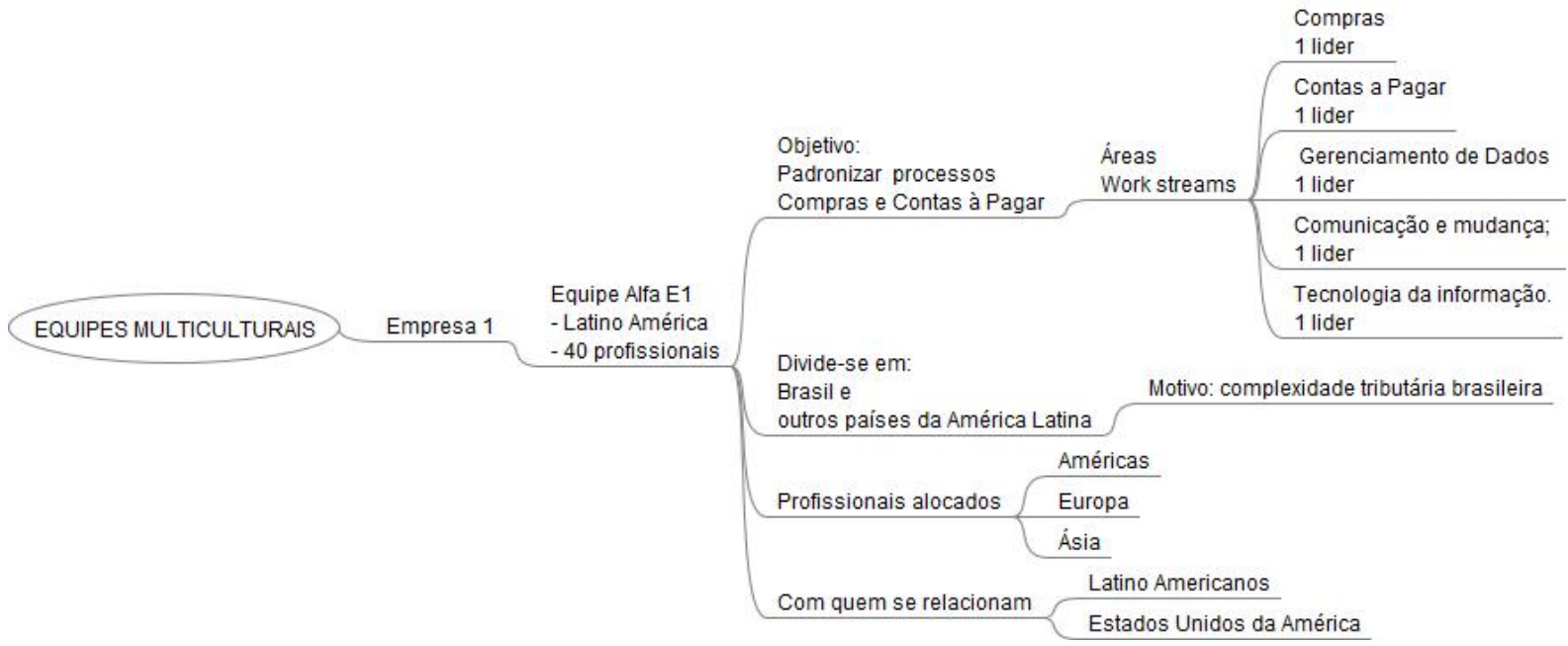

Figura 5. Caracterização da Equipe 1 da Empresa Alfa

Fonte: dados da pesquisa.

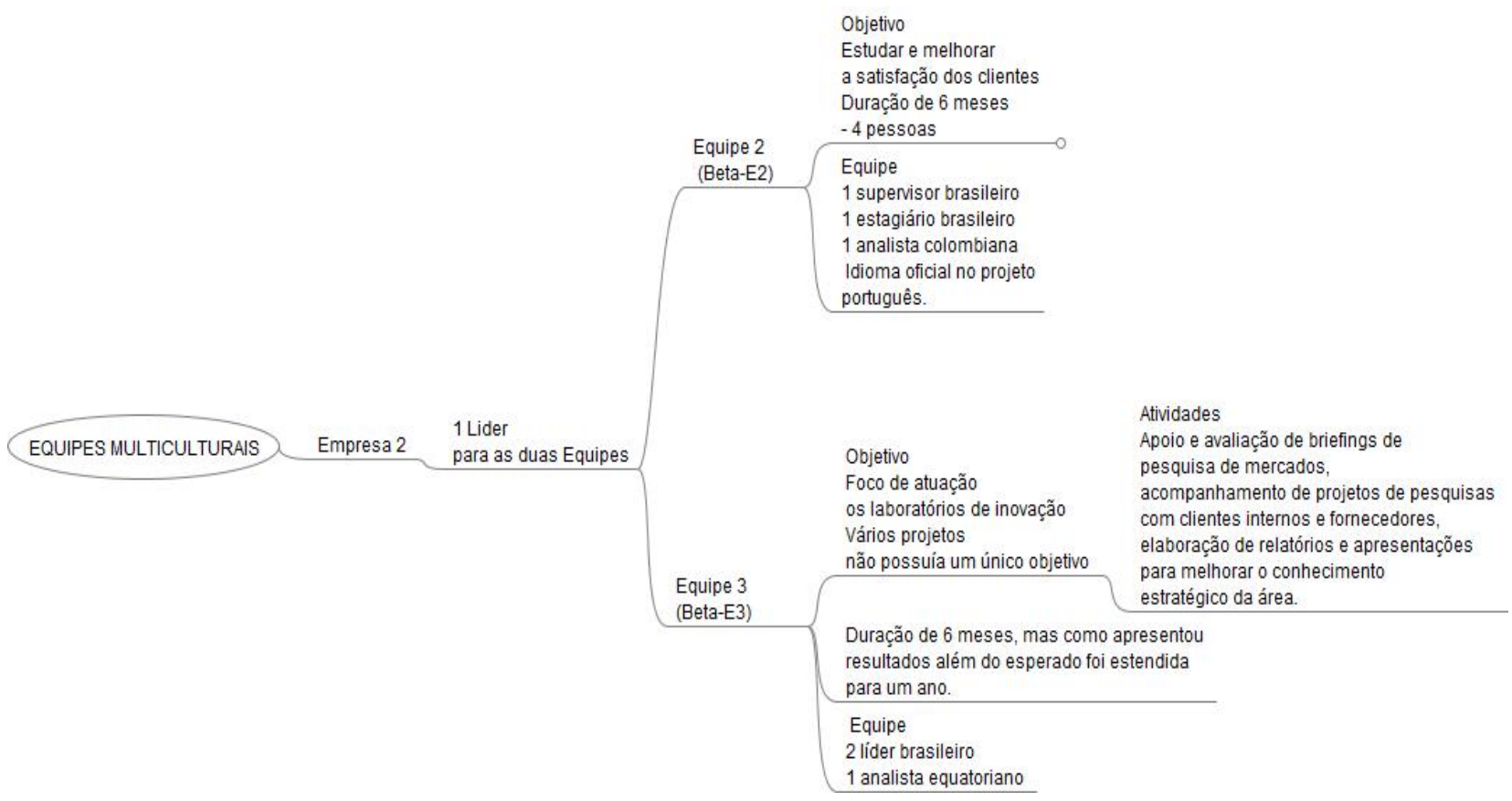

Figura 6. Caracterização das Equipes 2 e 3 da Empresa Beta

Fonte: dados da pesquisa.

Como é possível observar, ao analisar as figuras supracitadas, as equipes são diferentes em termos de número de membros, tempo de atividade, objetivo e tipos de projetos. Isto proporcionou uma análise mais abrangente das categorias: processo de liderança, influência da cultura nacional e processo de aprendizagem organizacional. 


\subsection{O processo de liderança das equipes multiculturais}

A entrevistada Beta-E2-6, assim como o entrevistado Beta-E3-7, relata que a personalidade do líder da equipe multicultural foi importante para guiar como a equipe agiria em conjunto. Beta-E2-6 acredita que o líder é capaz de influenciar muito na maneira como as pessoas agem conjuntamente e, dessa forma, o fato do líder ser paciente e bom ouvinte, permitindo que todos conseguissem expor suas opiniões para chegar a uma solução em conjunto, fez com que a equipe tivesse poucos problemas relacionados às opiniões divergentes e criasse um ambiente de tranquilidade para que todos fossem ouvidos.

O entrevistado Beta-E3-7 relata que os líderes que ele teve durante sua experiência com a equipe multicultural foram muito importantes para criar um ambiente seguro, com abertura para novas ideias e desenvolvimento de atividades de maneira autônoma. Os líderes trabalhavam para dar os parâmetros de desempenho e discutiam juntamente com ele os objetivos que deveriam ser atingidos e o deixavam livre para que fizesse da maneira que ele julgasse ser a melhor.

Segundo o entrevistado Beta-E2/3-5, líder das equipes E2 e E3, para que as equipes tenham ideias de forma ordenada, o papel do líder é crucial no esforço de entender a particularidade das pessoas, principalmente, quando se trata de equipes culturalmente diversas e depois unir os integrantes com objetivos e propósito claros. Isso faz com que os indivíduos que, incialmente, eram diferentes e sem interesses em comum trabalhassem bem juntos e conseguissem entregar um bom resultado. Ele comentou que, para criar um ambiente seguro para se opinar, ele busca (assim como o entrevistado Alfa-E1-4 que também é líder de equipe) escutar o que seus liderados têm a dizer e ponderar quando há opiniões distintas. Porém, ele acredita que há pessoas que, naturalmente, são mais seguras para mostrar seus pontos de vista e diz que cabe ao líder dar espaço e voz aqueles que estão com postura mais observadora ou coadjuvante na equipe.

O entrevistado Beta-E2/E3-5 explica que, durante a gestão das duas equipes, buscava criar um ambiente e interações informais e ele relaciona isto ao fato de sempre liderar equipes compostas, majoritariamente, por jovens. Ele acredita que em ambientes informais os jovens são mais produtivos e conseguem expor suas ideias e opiniões melhor do que em ambientes mais rígidos e formais. 


\subsection{A influência da cultura nacional no trabalho das equipes}

Todos os integrantes entrevistados da Alfa-E1 consideram que a diversidade vivenciada na equipe foi estimulante para os resultados do projeto, seja a diversidade relacionada à faixa etária, sexo, religião ou experiência acadêmica. Estas diferenças provocam debates, tornando o ambiente mais dinâmico e propício às melhores ideias e resultados. Todos ressaltaram que a cultura brasileira tem impacto na maneira como eles trabalham em equipe, sendo uma forma mais descontraída, amigável com seus colegas de trabalho e, principalmente, mais comunicativa do que outras culturas, sendo visto como algo que afeta positivamente o grupo.

Todos os integrantes da Alfa-E1 acreditam que o ambiente é seguro para que todos exponham suas opiniões, o que foi destacado é que algumas pessoas possuem um cargo de suporte ao projeto e por isso, em reuniões ficam mais retraídas ou também pela barreira linguística que existe, apesar de ser um pré-requisito para o projeto falar inglês ou espanhol. Esta barreira, segundo os entrevistados, é real e pode fazer com que um membro não se expresse muito durante reuniões, o entrevistado Alfa-E1-3 pondera que a liderança deixa claro que ferramentas para tradução e o chat podem ser utilizadas durante as teleconferências.

A entrevistada Beta-E2-6 destaca que as questões ligadas à diversidade mais marcantes para ela na sua equipe estavam relacionadas à idade (os colegas eram mais experientes do que ela); gênero; e à cultura nacional, formando um ambiente mais heterogêneo, mesmo se tratando de uma equipe pequena, e vê isso como sendo positivo para o resultado. Ela não observou muita diferença entre sua cultura (colombiana) e a brasileira, entende que os latinos compartilham muitas características e o traço da cultura colombiana mais evidente durante sua estada no Brasil foi não temer expor sua opinião.

Segundo Beta-E3-7, a diversidade afetou de maneira positiva apenas e as diferenças mais percebidas por ele estavam ligadas à idade e ao fato dele ser equatoriano, sendo assim, relata que diante de barreiras e divergências, os membros da equipe sempre conversaram e cada indivíduo expunha suas opiniões para, então, chegar a um consenso do que seria melhor aplicar. Sobre diferenças culturais, ele disse que o brasileiro é mais comunicativo do que o equatoriano e acredita que a característica de ser mais direto foi algo que marcou o início de sua experiência, mas que foi se adaptando e incorporando ao seu cotidiano a maneira de se comunicar e brincar do brasileiro.

O entrevistado Beta-E2/E3-5 não enxerga muitas diferenças culturais entre brasileiros e outros latinos, ele acredita que as culturas são parecidas e que povos latinos são 
comunicativos e abertos às discussões. Contudo, ele reconhece que há diferenças e que em suas equipes busca ambientes heterogêneos, principalmente com pessoas jovens, pois acredita que essa diversidade é positiva para o grupo e o resultado sempre é melhor em ambientes culturalmente diversos.

Três dos quatro entrevistados da Alfa-E1 classificou a comunicação da equipe como sendo informal, apontando esta informalidade como saudável e positiva para o desempenho do projeto. O idioma mais falado pela equipe é o espanhol, apesar disso o inglês é o idioma oficial com todos os materiais tendo uma versão em inglês. Não são todos os participantes que dominam o idioma espanhol, contudo, o entrevistado Alfa-E1-3 ressaltou que é possível que todos interajam com auxílio de softwares e aplicativos tradutores, por exemplo. A comunicação da Alfa-E1 feita de maneira virtual, que ocorre no dia a dia, ocorre por meio de e-mails, teleconferências e reuniões realizadas via Skype e chat; e face a face que tem como objetivo acelerar etapas que precisam ser realizadas em grupo. Os momentos face a face são tidos como necessários para construção de espírito de equipe e permitir maior proximidade entre os membros da equipe.

Por se tratar de uma equipe pequena e que todos estavam presentes fisicamente no mesmo local a maior parte do tempo, a entrevistada Beta-E2-6 relata que a comunicação era quase totalmente face a face, com reuniões presenciais e alguns e-mails, com interação bem informal. E, apesar da equipe não ter tido problema, ela reconhece que isso pode apresentar um risco por não ter muitos registros do que acontece e ao que era requerido pelos clientes. Após voltar para a Colômbia, a entrevistada continuou trabalhando à distância com esta equipe, então, a interação passou a ser totalmente virtual e de acordo com ela, essa interação é mais difícil por ter que lidar com a comunicação verbal e a não verbal que, muitas vezes, não é facilmente decifrada. Dessa forma, as mensagens e e-mails tinham duas ou mais formas de serem interpretados e causavam problemas de comunicação.

O entrevistado Beta-E3-7 relata que o terceiro participante da equipe não estava presente fisicamente no mesmo local que ele e o entrevistado Beta-E3-5 ficavam, no entanto, tratava-se da mesma cidade, então, eram utilizadas ferramentas como e-mails e alguns chats para a comunicação diária cotidiana. Quando se tratava de uma etapa importante do projeto, eles se deslocavam para se encontrar pessoalmente. Já entre o Beta-E3-5 e Beta-E3-7, a comunicação era totalmente face a face, com reuniões presenciais e, havendo dúvidas, o líder sempre estava aberto para questionamentos.

O idioma oficial da Beta-E2 era o português, a entrevistada Beta-E2-6 conta que chegou ao Brasil com nível intermediário do idioma, mas para conversar ela era mais devagar 
que as outras pessoas. Os dois primeiros meses no Brasil foram de adaptação com o idioma e com os sotaques existentes. Assim como na Beta-E2, na Beta-E3 o idioma oficial para comunicação era o português, o entrevistado Beta-E3-7 relata que chegou no Brasil para a experiência sem nenhuma base de português, então ele tentava se comunicar utilizando o espanhol e o "portunhol" e, algumas vezes, o inglês. Ele conta que demorou, aproximadamente, três meses para se adaptar bem ao idioma, mas que durante essa fase o mais complicado era se comunicar com o restante da empresa, já que dentro da equipe ele conseguia se comunicar bem, pois os integrantes tinham pelo menos o inglês como segundo idioma, o que não era uma realidade dentro da empresa como um todo.

\subsection{Aprendizado da equipe multicultural}

A ferramenta utilizada para registro do conhecimento na Alfa-E1 é um SharePoint, que é uma plataforma localizada na intranet da companhia que tem como função registrar e compartilhar os conhecimentos de cada área e de cada projeto realizado. Qualquer empregado da empresa tem acesso para leitura dos documentos compartilhados nesta plataforma, lá estão os principais arquivos e as análises mais significativas, divididas por regiões e por work streams. Nesta ferramenta estão documentadas e catalogadas todas as etapas do projeto, o que foi requisitado pela equipe, o que foi aceito no projeto, e também o que não foi aceito juntamente com os principais acontecimentos, análises e fluxos de cada fase. O projeto conta com uma equipe de comunicação e esta é a responsável pelo gerenciamento da mudança e treinamentos demandados pelo projeto. Também é responsabilidade desta equipe certificar-se que o projeto seja documentado e registrado.

A entrevistada Beta-E2-6 comenta que foi gerado conhecimento sobre como desenvolver uma solução para os clientes, sobre fornecedores, construção de plano de ação e convencimento estruturado pela equipe sobre investimentos importantes, como a internacionalização da empresa Beta. No período em que esteve no Brasil, ela conseguiu aprender muito sobre a cultura brasileira, assim como seus colegas de trabalho aprenderam sobre a cultura colombiana na execução de determinados processos.

A empresa Beta não possui nenhuma ferramenta específica de gestão do conhecimento e o conhecimento e processo de aprendizado foram registrados por meio de e-mails trocados, em apresentações realizadas com os clientes e superiores, por pequenos manuais e catálogos com dados dos fornecedores que foram desenvolvidos em conjunto pelos membros da equipe. A entrevistada Beta-E2-6 relata que isto era acessível apenas para os membros da equipe 
multicultural, não havendo forma de tornar estas informações disponíveis para os outros empregados da empresa.

No que diz respeito aos treinamentos oferecidos, a entrevistada comenta que sentiu falta, por ser estrangeira, de treinamento e um acompanhamento mais próximo por parte da empresa com questões rotineiras sobre o modo de vida na cidade. Ademais, ela ressalta que gostaria que a empresa tivesse proporcionado uma apresentação sobre as empresas que faziam parte do grupo e as áreas funcionais com quem ela interagia.

Como a Beta-E3 não trabalhava em apenas um projeto e sim em vários, o entrevistado Beta-E3-7 coloca como um dos grandes aprendizados desta equipe a comunicação interna. No início isto era algo difícil para eles, era preciso mais de um recurso para que a mensagem fosse passada, muitas vezes eles tentavam explicar em português misturado com espanhol, ou então, o inglês e uso de recursos visuais como desenhos, por exemplo. Contudo, a equipe apresentou resultados além do esperado, resultando em um plano de investimento. Dessa forma, o know-how de como construir um plano deste tipo foi algo adquirido pela equipe com a aquisição do conhecimento técnico também ao longo da experiência coletiva.

Assim como a Beta-E2, a Beta-E3 não possuía nenhuma ferramenta de compartilhamento de conhecimentos, conforme o entrevistado Beta-E3-7 tudo o que acontecia nos projetos e na equipe ficava registrado apenas nos e-mails trocados e apresentações realizadas. Por isso, não havia como outras áreas e outras empresas do grupo saberem e utilizarem os conhecimentos e aprendizado obtidos de modo a beneficiar outros projetos.

A empresa promoveu alguns treinamentos técnicos e uma organização parceira intermediou a experiência intercultural realizando treinamentos culturais com estrangeiros que estavam no país. Esses treinamentos tinham mais o intuito de socialização e criação de laços de amizade entre esses estrangeiros, não havia uma estrutura formal nos treinamentos. Apesar dessa observação, ele diz que não sentiu falta de nenhum treinamento e ele relaciona isso com o fato de preferir viver e experimentar as diferenças e dificuldades na prática.

\subsection{Resultados alcançados pelas equipes multiculturais e discussão}

Em relação aos resultados para os profissionais envolvidos em uma equipe multicultural, todos os entrevistados da Alfa-E1 ressaltaram que o benefício de se viver tal experiência é a de conhecer realidades diferentes dentro de uma mesma empresa, conhecer outras culturas e, principalmente, a visibilidade tanto internamente quanto externamente à organização que participar de um projeto multicultural traz. Convergiram quanto aos 
benefícios que este tipo de equipe traz para a empresa, sendo que as maiores vantagens estão em conseguir documentar como cada país faz os seus processos e chegar a um denominador comum.

A entrevistada Beta-E2-6 afirma que a sua maneira de trabalhar em equipe mudou bastante desde a sua experiência com Beta-E2 que a tornou uma pessoa muito mais paciente e mais tolerante à diversidade e ao que é diferente da realidade que ela conhece. Estes dois fatores em conjunto fazem com que ela acredite que trabalhe melhor em equipe do que antes.

O entrevistado Beta-E3-7 relata que a equipe multicultural mudou sua maneira de se comportar no trabalho também, visto que ele se tornou mais comunicativo depois desta experiência e relaciona isto com a experiência no Brasil, pois os brasileiros são mais comunicativos e abertos ao novo do que os equatorianos e ele incorporou estas características à sua maneira de trabalhar contribuindo para melhorar sua participação no trabalho no trabalho em equipe.

O entrevistado Beta-E2/E3-5 pontua que a maior mudança depois de viver as experiências em diferentes equipes multiculturais foi comportamental, ele afirma que se tornou mais tolerante e paciente com o diferente após liderar as duas equipes. Depois destas experiências interculturais ele prioriza em suas equipes pessoas que tenham visões diferentes e com perfis distintos para que se tenha, mesmo em pequena escala, equipes plurais e heterogêneas, ressalta que prefere pessoas que discutam e instiguem os outros a repensar, para assim chegar a melhor solução possível.

Assim como a Alfa-E-1, os da Beta-E2 e Beta-E3 afirmaram que a vantagem, do ponto de vista profissional, de se viver uma experiência multicultural é de conhecer outras realidades e aprender novas maneiras de fazer processos. Ademais, o entrevistado Beta-E3-7 relata que a sua experiência na empresa Beta foi muito positiva para sua carreira, e por isso ele decidiu morar no Brasil definitivamente. Ele relaciona isso ao quanto sua maneira de trabalhar foi transformada positivamente depois da sua participação na equipe multicultural. Ele ressalta que as equipes multiculturais possuem, muitas vezes, profissionais excelentes com experiências singulares e a rede de contatos que se constrói neste tipo de equipe é muito grande e rica, e o fator networking foi crucial no que diz respeito ao rumo que sua vida profissional tomou após o fim do projeto da equipe.

Quanto às vantagens que as equipes multiculturais apresentaram para a empresa Beta, todos os entrevistados disseram que foram vantagens financeiras, já que nas duas equipes os estrangeiros entregaram trabalhos que resultaram na sua internacionalização e em um plano 
de investimento, ambos os projetos equivaleram a uma grande parcela do orçamento disponível.

A partir das análises realizadas foi possível perceber a existência de alguns pontos de convergência e de divergência entre as respostas dos entrevistados das 3 equipes e com a literatura sobre equipes multiculturais no que concerne a questões de liderança, cultura nacional e processos de aprendizagem.

Sobre o processo de liderança, todos os entrevistados das três equipes reconheceram o papel fundamental da figura do líder dentro de uma equipe multicultural, direcionando o grau de formalidade ou informalidade da interação entre os integrantes, dando os parâmetros de desempenho e os objetivos a serem alcançados e, principalmente, para mediar conflitos. Levando isso em conta e com base nas respostas dos entrevistados, verificou-se que nas três equipes a liderança é personificada em um único indivíduo, retratando a combinação entre atividades focadas e autoridade vertical para tomada de decisão, tendo como base a tipologia de Zander e Butler (2010). Isto pode ser explicado, pelo menos em parte, na empresa Alfa pela estruturação da equipe levando-se em conta o número de profissionais envolvidos, a orientação mais técnica e a definição do escopo do projeto. Na empresa Beta, a explicação se dá pelos objetivos mais pontuais e específicos das equipes, pela juventude dos seus membros e, também, pelo pouco tempo de existência das equipes. Sendo que uma delas já não existe mais formalmente, pois já cumpriu seu objetivo focado em um projeto específico.

Em relação à segurança, para que as equipes multiculturais tenham alto desempenho e consigam aprender de fato, segundo Garvin, Edmondson e Gino (2008), é preciso oferecer um ambiente seguro psicologicamente, onde os indivíduos não sintam medo de compartilhar e discordar e que os participantes apreciem as diferenças. Apesar de todos os entrevistados pontuarem que se sentiam seguros para expor suas opiniões, os líderes ponderaram que existem indivíduos que são mais retraídos e não se sentem confortáveis em exibir seus pontos de vistas. Além disso, merece destaque que a questão de segurança surgiu também relacionada com o domínio (ou falta dele) com relação ao idioma sendo apontado como prejudicial para a produtividade e resultado da equipe.

Alguns pontos levantados pelos entrevistados se relacionam com alguns traços da cultura e da gestão brasileira mostrados por Hofstede (1984) e Chu (2011), que mostram a influência da cultura nacional. Como exemplos, o desconforto dos brasileiros para expor opiniões em ambientes diferentes daqueles em que estão acostumados e com relações mais horizontalizadas como apontado nos traços sobre distância do poder e postura de expectador. Outro ponto que se pode notar é o traço da feminilidade, pois os brasileiros gostam de ter 
relacionamentos próximos e informais juntos aos seus colegas de trabalho e superiores causando estranheza em seus colegas estrangeiros, pelo menos no primeiro momento quando dos contatos e convivência inicial. Diante disso, é possível dizer que a necessidade de proximidade e relação interpessoal informal sejam formas condicionadas de superar a barreira (existente ou não) da hierarquia e das distâncias entre os espaços de poder.

No que se refere à comunicação intercultural, os entrevistados relataram enfrentar dificuldades e ciência da sua complexidade que vai além de questões linguísticas, como já apontado por Adler e Gundersen (2008). Houve falhas de entendimento principalmente no início da formação das equipes, mas com o convívio e também o auxílio de programas de tradução, uso da criatividade dos indivíduos com diferentes recursos para se fazer compreender como desenhos e mediação dos líderes foi possível driblar ou, pelo menos, diminuir esta barreira.

Houve situações conflituosas enfrentadas por todas as três equipes, conforme argumentaram Adler e Gundersen (2008) e Stahl et al (2010), por se tratar de equipes diversas isso pode causar conflitos de concepções culturais, falta de entendimento do significado dado aos fatos e situações afetando o entrosamento e a produtividade. Apesar disso, tais situações resultaram em saldo positivo na opinião dos entrevistados. O papel de liderança formal foi citado pelos respondentes como fundamental para gerir estas situações a fim de minimizar o estresse e auxiliar na negociação por busca de soluções. Há que se considerar, também, a questão de menor distanciamento de contextos culturais, sendo as culturas nacionais envolvidas consideradas como de alto contexto, confomre Hall (1976), o que pode ajudar a explicar a menor dificuldade de comunicação, diferentemente do que poderia ocorrer na relação entre culturas de alto e baixo contexto.

Sobre a gestão do conhecimento das empresas estudadas e o processo de aprendizagem organizacional, verificou-se que apesar de identificarem diferenças culturais, os membros das equipes foram capazes de dialogar e compartilhar seu conhecimento. Como já apontado por Blanche e Dupuis (2019), alguns encontros culturais que produzem sinergia entre os participantes são aqueles onde o foco não reside nas diferenças culturais, mas sim nos pontos comuns e nos pontos de contato que mobilizam diferentes identidades.

Também é importante ressaltar que as organizações existem independentemente das pessoas, mas são os indivíduos que possuem o conhecimento e a habilidade de solucionar problemas. Dessa forma, surge a necessidade de se criar a memória organizacional para assim diminuir a relação de dependência das empresas em relação aos indivíduos que ali trabalham. A empresa Alfa está criando a memória dos projetos de equipes multiculturais usando uma 
ferramenta formal, em contrapartida, a empresa Beta não possui nenhum instrumento para a gestão do conhecimento, portanto as equipes Beta E2 e Beta E3 não registraram formalmente sua experiência e o conhecimento gerado.

Mesmo entendendo ser uma prática complexa, Silva e Burger (2017) apontam a necessidade de se gerenciar o conhecimento adquirido para gerar vantagem competitiva e impulsionar a inovação, nesse sentido, a empresa Beta pode estar deixando escapar oportunidades de avançar nos seus processos de trabalho e soluções para seus problemas e de seus clientes, visto que o conhecimento adquirido pelos membros de suas equipes multiculturais não tem sido capitalizado pela empresa. Ambas as empresas podem melhorar em seus processos de geração de conhecimento e aprendizagem organizacional e com isso fomentar uma cultura de inovação e forma criativa de solução de problemas.

Apesar dos pontos de melhoria a se alcançar, é inegável que foram obtidos diferentes ganhos e benefícios com o trabalho das equipes multiculturais, como apontado pelos entrevistados, corroborando a literatura (Adler \& Gundersen, 2008; Bueno \& Freitas, 2018, por exemplo) onde são observados que os benefícios não são só para a organização (vistos nas equipes estudadas na forma de superação das expectativas com relação aos resultados), mas os participantes também conseguem experiência e projeção nas suas carreiras. E os respondentes têm ciência deste valor da diversidade, também citado por Mannix e Neale (2016), observada em diferentes momentos das entrevistas, seja no reconhecimento do ambiente heterogêneo como positivo para o desempenho das equipes e para si mesmos do ponto de vista da experiência e aprendizado, seja também na perspectiva da carreira profissional. Ademais, é importante ressaltar que duas das três equipes multiculturais estudadas apresentaram resultados além do esperado por seus gestores. As equipes multiculturais quando bem gerenciadas são altamente criativas, inovadoras e estratégicas, nos casos estudados a equipe Beta E2 resultou em um plano de internacionalização da empresa e a equipe Beta E3 apresentou em um plano de investimento em startups.

\section{Considerações Finais}

O objetivo deste trabalho foi analisar, em duas empresas multinacionais, os desafios de três equipes multiculturais de trabalho tendo como parâmetros as questões sobre liderança, a influência da cultura nacional e o processo de aprendizagem organizacional. Observou-se que, neste grupo pesquisado, o tipo predominante é a liderança única e vertical que apresentou ganhos, mas é preciso trabalhar para a formação e atuação de diferentes lideranças em equipes 
multiculturais que possam ser mais adequadas à cada situação e objetivos, e que irão influenciar nos resultados obtidos, como a liderança rotativa e compartilhada que suportam melhor a pluralidade que se tem em ambientes multiculturais.

Também foi evidenciada a influência da cultura nacional no trabalho de equipes multiculturais, com o destaque de algumas características da cultura brasileira que interferiram, de algum modo, na interação e execução das atividades das equipes. É importante salientar a necessidade da manutenção de um ambiente de confiança, principalmente para os brasileiros no que diz respeito a sentirem-se à vontade para participar e emitir opiniões divergentes, pois isto afeta a questão de segurança quanto à empregabilidade, uma vez que pode gerar conflitos. Assim, deve-se acompanhar mais de perto situações onde o conflito é suprimido ou velado para ajudar tanto nos processos de liderança e aprendizagem como no desenvolvimento dos profissionais e da organização.

Outro ponto relevante, fruto desta pesquisa, foi a importância do registro do conhecimento adquirido com as atividades das equipes multiculturais e as diferentes formas que podem ser utilizadas para impulsionar a aprendizagem e a melhoria dos processos organizacionais. Cabe ressaltar os relevantes ganhos e benefícios que as equipes estudadas trouxeram, ao longo do tempo, para as empresas e também para os seus membros. Tais resultados superaram as expectativas iniciais, tanto individualmente como em equipe e ajudaram a projetar a carreira de seus membros evidenciando que esta experiência tende a ser muito enriquecedora para os profissionais envolvidos como também para as organizações.

Este artigo contribui com a literatura e a prática gerencial por não ter como foco de análise elementos pontuais de contexto ou processo, mas, sim, trata-los de forma interrelacionada e suas dinâmicas, levando-se em conta os resultados obtidos pelas equipes multiculturais, pelos indivíduos e empresas. Tal contribuição pode auxiliar na articulação, implementação e desenvolvimento de equipes multiculturais nas empresas tendo por parâmetros o processo de liderança e seus diferentes tipos, os aspectos culturais e como podem afetar os membros das equipes, bem como, definir os melhores instrumentos e processos para a gestão do conhecimento e aprendizado gerados. Assim, verificou-se que estes elementos são catalisadores, isto é, aceleram o amadurecimento das equipes e dos seus líderes.

O estudo apresentou limitações quanto ao tamanho da amostra, já que as equipes Beta E2 e Beta E3 são da mesma empresa e com poucos membros. Entretanto, ele deve ser somado a outras pesquisas sobre o tema para formar um framework mais abrangente, uma vez que as 
organizações multinacionais, no Brasil, apresentam restrições para pesquisas envolvendo profissionais de outros países.

Para pesquisas futuras, sugere-se estudar a opinião de um maior número de estrangeiros em equipes multiculturais onde trabalham brasileiros. Também seria importante estudar as mudanças de configuração de equipes multiculturais e como os novos membros são inseridos na dinâmica de trabalho destas equipes. Outra sugestão é aprofundar a análise integrada entre elementos contextuais e elementos processuais para auxiliar a preparação e também a avaliação de equipes multiculturais de trabalho.

\section{Referências}

Adler, N. J.; \& Gundersen, A. (2008). International dimensions of organizational behavior. 5a ed. New York: South Western.

Algesheimer, R., Dholakia, M.; \& Gurau, C. (2011). Virtual team performance in a highly competitive environment. Group \& Organization Management, 36 (2), p. 161-190.

Bardin, L. (2009). Análise de Conteúdo. Lisboa: Edições 70.

Bento, J.; Urpia, A.; Bortolozzi, F.; \& Massuda, E. (2017). Nível de implantação e alcance das práticas da gestão do conhecimento em base tecnológica e funcional de organização educacional. Revista Alcance, 24 (2), p. 243-257.

Blanche, C.; \& Dupuis, J. P. (2019). From The Hague to Paris to Montreal: knowledge transfer and cultural synergy in a multicultural organization. International Journal of Cross Cultural Management, 19(1), pp. 27-46.

Bueno, J.; \& Freitas, M. (2015). As equipes multiculturais em subsidiárias brasileiras de multinacionais: um estudo de casos múltiplos. Organizações \& Sociedade, 72 (1), p. 15 34.

Bueno, J. M., \& Freitas, M. E. (2018). Representações Sociais no Contexto Intercultural: O Cotidiano de Três Subsidiárias Brasileiras. Cadernos EBAPE.BR, 16(1), 101-118.

Cheng, C.; Chua, R.; Morris M.; \& Lee, L. (2012). Finding the right mix: How the composition of self-managing multicultural teams' cultural value orientation influences performance over time. Journal of Organizational Behavior. p. 389-411.

Chevrier, S. (2009). Is national culture still relevant to management in a global context? The case of Switzerland. International Journal of Cross-cultural Management, 9(2), pp. 169184. 
Chu, R. (2011). Modelo Contemporâneo da Gestão a Brasileira. São Paulo: Cengage Learning.

Connaughton, S.; \& Shuffler, M. (2007). Multinational and multicultural distributed teams. Small Group Research, 38 (3), p. 387-412.

Davoine, E.; \& Barmeyer, C. (2015). Intercultural competence of binational pairs as a supporting factor of negotiated culture in binational organisations: An analysis of the French-German case of Alleo. In: EGOS COLLOQUIUM, Atenas, Grécia, Anais.

Dewes, J. (2013). Amostragem em bola de neve e respondent-driven sampling: uma descrição dos métodos. p 53 f. Trabalho de Conclusão de Curso (Graduação) - Curso de Estatística, Universidade Federal do Rio Grande do Sul, Porto Alegre.

Fleury, M.; Fleury, A. (2001). Construindo o conceito de competência Revista de Administração Contemporânea, 5(1), p. 183-196.

Garvin, D.; Edmondson, A.; \& Gino, F. (2008). Is yours a learning organization? Harvard business review, 86 (3), p. 1-10.

Hall, E. T. 1976. Beyond culture. New York: Anchor Books/Doubleday.

Heinz, K. (2014). Multicultural team conflict management. Securitologia, 1(19), p.117-128.

Hofstede, G. (1984). Culture's consequences: International differences in work related values. 5ed. Beverly Hills: Sage.

Krumm, S., Terwiel, K.; \& Hertel, G. (2013). Challenges in norm formation and adherence: The knowledge, skills, and ability requirements of virtual and traditional cross-cultural teams. Journal of Personnel Psychology, 12 (1), p. 33-44.

Leinonen, P.; Jarvela, S.; \& Hakkinen, P. (2005). Conceptualizing the awareness of collaboration: a qualitative study of a global virtual team. The Journal of Collaborative Computing and Work Practices, 14(1), p. 301-322.

López, S.; Peón, J.; \& Ordás, C. (2015). Organizational learning as a determining factor in business performance. The Learning Organization, 12(3), p.227-245.

Mannix, E.; \& Neale, M. (2005). What differences make a difference? The promise and reality of diverse teams in organizations. Psychological Science in the Public Interest, 6 (2), p. 31-55.

Markovic, D; Radovic-Markovic, M.; \& Minovic, J. (2015). A new virtual team competence defining model. Economic Research Istrazivanja. 28(1), p. 1034-1045.

Pudelko, M., Carr, C., \& Henley, J. (2007). Globalization and its effects on international strategy and cross-cultural management. International Studies of Management and Organization, 36(4), pp. 3-8.

Revista de Gestão e Secretariado (GeSec), São Paulo, SP, 11(2), maio/ago., 2020, p. 164-189. 
Schein, H. (2009). Cultura organizacional e liderança. São Paulo: Atlas.

Silva, T.; \& Burger, F. (2018). Aprendizagem organizacional e inovação: contribuições da Gestão do Conhecimento para propulsionar um ambiente corporativo focado em aprendizagem e inovação. Navus, 8(1), p.7-19.

Stahl, G.; Maznevski, M.; Voigt, A.; \& Jonsen, K. (2010). Unraveling the effects of cultural diversity in teams: A meta-analysis of research on multicultural work groups. Journal of International Business Studies, 41(4), p. 690-709.

Strauhs, F.; Pietrovski, E.; Santos, G.; Carvalho, H.; Pimenta, R.; \& Penteado, R. (2012). Gestão do Conhecimento nas Ogranizações. Curitiba: Ayamará Educação.

Takeuchi, H.; \& Nonaka, I. (2008). Gestão do Conhecimento. Porto Alegre: Bookman.

Vinuto, J. (2014). A amostragem em bola de neve na pesquisa qualitativa: um debate em aberto. Temáticas, 22, (44), pp. 203-220, ago/dez.

Zander, L.; \& Butler, C. L. (2010). Leadership modes: success strategies for multicultural teams. Scandinavian Journal of Management, 26, p. 258-267.

Submetido em: 24.01.2020

Aceito em: $\quad 24.03 .2020$ 